\title{
A pseudoepidemic of Mycobacterium chelonae infection caused by contamination of a fibreoptic bronchoscope suction channel
}

\author{
H-C. Wang, Y-S. Liaw, P-C. Yang, S-H. Kuo, K-T. Luh
}

A pseudoepidemic of Mycobacterium chelonae infection caused by contamination of a fibreoptic bronchoscope suction channel. H-C. Wang, Y-S. Liaw, P-C. Yang, S-H. Kuo, K-T. Luh. @ERS Journals Ltd 1995.

ABSTRACT: An unusual increase in the frequency of isolation of Mycobacterium chelonae subspecies chelonae from specimens of bronchial washings was found between September and December 1992 in National Taiwan University Hospital.

During this period, a total of 123 patients underwent fibreoptic bronchoscopy with an Olympus P20. Seventy six patients had bronchial washing for bacteriological study and cytological examination. Acid-fast bacilli were found in 21 patients, in 18 of whom Mycobacterium chelonae were isolated from bronchial washing cultures. Eight patients were treated as mycobacterial infected, because of the presence of unexplained pulmonary lesion, positive acid-fast stain and culture for Mycobacterium chelonae. Diagnosis of lung cancer was delayed in one patient because of the initial negative cytological study and positive bacterial culture. The fibreoptic bronchoscope was disinfected by automated washing machine (EW-20, Olympus) using $2.3 \%$ glutaraldehyde according to a standard protocol. From a survey to search for possible sources of contamination, they were identified at the suction channel of four different bronchoscopes. This episode proved to be a pseudoepidemic. The contamination was controlled by extensive suction and rinsing of the channel with $70 \%$ alcohol immediately after disinfection by the automated bronchoscope disinfection machine.

This study shows that, despite using the disinfection machine, the suction channel could still be contaminated with Mycobacterium chelonae. This may cause diagnostic confusion and unnecessary antimycobacterial treatment.

Eur Respir J., 1995, 8, 1259-1262.
Dept of Internal Medicine and Laboratory Medicine, National Taiwan University Hospital, Taipei, Taiwan, Republic of China.

Correspondence: P-C. Yang

Dept of Internal Medicine

National Taiwan University Hospital

No. 7 Chung-Shan South Road

Taipei,

Taiwan

Keywords: Bronchoscopic suction channel Mycobacterium chelonae

pseudoepidemic

Received: December 191994

Accepted after revision April 161995
Mycobacterium chelonae, a rapid growing mycobacterium, is a member of the Runyon group IV of mycobacteria which are commonly isolated from water and soil. They are generally regarded as commensals in human [1]. M. chelonae most frequently causes cutaneous and soft tissue infection following accidental trauma or surgery, in a variety of clinical conditions [2]. Arthritis, lymphadenitis, pericarditis, mediastinitis and pulmonary diseases have all been implicated [3,4]. Rarely, disseminated diseases in immune compromised patients have also been reported [5]. Most clinical disease is sporadic and community-acquired, but nosocomial epidemics have been described. The possible source of outbreaks has been identified as exposure to nonsterile water, ice and inadequately disinfected, reusable instruments [6-8].

An unusual increased frequency of isolation of $M$. chelonae from specimens of bronchial washings was noted between September and December 1992, in National Taiwan University Hospital. During this period, M. chelonae was isolated from 18 patients. All 18 isolates of $M$. chelonae came from specimens of bronchial washing. This report describes the investigation of the outbreak, and the measure taken to eradicate the source of contamination.
Materials and methods

National Taiwan University Hospital is a 2,000 bed medical centre in which some 400 bronchoscopic examinations are performed annually. Bronchoscopy is performed via the transnasal route with four flexible fibreoptic bronchoscopes (Olympus BF, P20). Bronchial washings are regular diagnostic procedures for aspiration of bronchial fluid for microbiological and cytological study. The bronchial washings are carried out with injection of $30 \mathrm{~mL}$ normal saline through a fibreoptic bronchoscope to the affected bronchus. Specimens are then aspirated and collected in a sterile plastic tube for cytological and microbiological examinations, which include Gram stain, acid-fast stain, common bacterial and mycobacterial cultures. The bronchoscope is cleaned and disinfected immediately after each use.

The routine cleansing and disinfection procedure is as follows: the outside of the bronchoscope is immersed in tap water and washed with gauze; suction valves are dismantled and, together with the suction channel, are also brushed clean with tap water. The instrument is then disinfected in an automated washing machine (EW-20, 
Olympus) using $2.3 \%$ glutaraldehyde (Cidex, JohnsonJohnson) as a disinfectant. The standard protocol to clean and disinfect bronchoscopes is as follows: initial cleaning with neutral detergent and water; disinfection by immersion in glutaraldehyde for $10 \mathrm{~min}$, followed by a final rinse in tap water. The automated washing machine was first used in June 1992. Prior to that time, the bronchoscopes had been manually disinfected. The disinfectant was replaced every 4 weeks. The machine itself was not disinfected routinely.

A review of the hospital's laboratory and bronchoscopic records from January 1990 until December 1993 was undertaken. Medical records of all patients with positive culture for $M$. chelonae were reviewed using a standardized questionnaire. Information was obtained on patient demographics, laboratory and pathology results, medical history, including prior hospitalizations and procedures, radiographic and physical examination findings at the time of specimen collection, history of smoking or alcohol use, history of prior mycobacterial exposure, disease, or treatment. Instrument disinfection procedures during the outbreak period were reviewed in detail.

A case-control study was performed to determine aspects of the bronchoscopy procedure that might be associated with $M$. chelonae pseudoepidemic. Cases were defined as patients, whose bronchial washing specimens yielded M. chelonae. Controls were randomly chosen from the bronchoscopy log book covering the 36 month period. They were patients who had bronchial washing specimens sent for mycobacterial culture that did not yield $M$. chelonae. Information about the physician performing the procedure, nurse assistant, date, age of disinfecting solution, and time elapsed since the bronchoscope was cleaned, was collected for all cases and controls.

A survey to search for a possible source of contamination was conducted by sampling the materials used during bronchoscopy and subsequent cleaning and disinfection of the bronchoscope. This included samples of local anaesthetic, 14 and 28 day old glutaraldehyde solution, hot and cold water taps in the room where bronchoscopes were cleaned, and swab from the disinfecting machine. Samples of rinsing fluid from four already disinfected bronchoscopes were also obtained. These specimens were decontaminated and concentrated for acid-fast smears, then inoculated onto Lowenstein-Jensen medium. All media were examined weekly for a total of 8 weeks. The colonies grown were further identified by subculture using Anti-Fast Set (Kyokuto, Japan) [9].

Patients were diagnosed as having $M$. chelonae pulmonary infection if they fulfilled the American Thoracic Society's (ATS) diagnostic criteria of nontuberculous mycobacterial lung disease, which include two or more specimens culture-positive for $M$. chelonae and exclusion of other reasonable causes of disease [10].

\section{Results}

Between January 1990 and December 1993, 25 cultures from 25 patients were positive for $M$. chelonae. Five of the 25 positive isolates were from corneal swabs, one from soft tissue pus, one from empyema, and 18 from bronchial washings. The positive cultures of specimens of all bronchial washings were clustered between September and December 1992. During this 4 month period, 123 patients underwent fibreoptic bronchoscopy. Seventy six patients had bronchial washing for bacteriological study and cytological examination. Acid-fast bacilli on smears were found in 21 patients and $M$. chelonae subspecies chelonae was recovered in the culture of bronchial washing in 18 patients. The mean age of the patients was 60 yrs (range, 45-79 yrs). The underlying diseases were lung cancer in three, lung cancer with pneumoconiosis in one, diabetes mellitus in one, and pulmonary tuberculosis with bilobectomy in one. The indications for bronchoscopy were chronic cough in seven, abnormal chest radiograph findings in 10, and haemoptysis in one. Radiographic findings are shown in table 1.

Table 1. - Clinical features of 18 patients whose culture of bronchial washing yielded M. chelonae

\begin{tabular}{cccccccc}
\hline $\begin{array}{c}\text { Case } \\
\text { No. }\end{array}$ & $\begin{array}{c}\text { Age } \\
\text { yrs }\end{array}$ & Sex & $\begin{array}{c}\text { Underlying } \\
\text { disease }\end{array}$ & $\begin{array}{c}\text { Acid-fast stain } \\
\text { of bronchial washing }\end{array}$ & $\begin{array}{c}\text { Radiographic } \\
\text { finding }\end{array}$ & $\begin{array}{c}\text { Sputum culture } \\
\text { mycobacteria }\end{array}$ & $\begin{array}{c}\text { Anti-TB } \\
\text { treatment }\end{array}$ \\
\hline 1 & 65 & F & Nil & + & Normal & - & - \\
2 & 53 & M & Nil & + & LUL Nodule & - & + \\
3 & 65 & M & Nil & - & Normal & - & + \\
4 & 55 & F & Nil & - & Rt B6 pneumonia & - & - \\
5 & 74 & M & Lung cancer & - & Rt B3 tumour & - & + \\
6 & 45 & M & Nil & - & LUL Nodule & - & + \\
7 & 66 & F & Nil & - & Normal & - & - \\
8 & 79 & M & Lung cancer & - & RUL lobectomy & - & + \\
9 & 69 & M & Nil & + & Normal & - & - \\
10 & 71 & M & Lung cancer & - & LUL tumour & - & - \\
11 & 60 & F & Nil & - & Pleural thickening & - & - \\
12 & 69 & M & Nil & - & RUL collapse & - & - \\
13 & 57 & M & TB, bilobectomy & - & Lobectomy & - & - \\
14 & 66 & M & Nil & - & RUL infiltration & - & - \\
15 & 45 & F & Nil & - & LUL nodule & - & + \\
16 & 71 & M & DM & - & RUL consolidation & - & + \\
17 & 71 & M & Lung cancer & - & LUL tumour & - & - \\
18 & 51 & M & Nil & - & Normal & - \\
\hline
\end{tabular}

TB; tuberculosis; DM; diabetes mellitus; M; male; F; female; LUL: left upper lobe; Rt: right; RUL: right upper lobe. 
The sputum cultures were all negative for mycobacteria. Eight patients were treated as mycobacterial infection initially because of the presence of unexplained pulmonary lesion, positive acid-fast stain and culture for M. chelonae from bronchial washings. Anti-tuberculous drugs were discontinued after a pseudoepidemic was confirmed. No patient met the ATS criteria for $M$. chelonae pulmonary disease. Diagnosis of lung cancer was delayed for 4 months in one patient because of the initial negative cytology study and positive bacterial culture. The case-control study did not show difference between the 18 case-patients whose bronchial washings yielded $M$. chelonae and the 30 control subjects whose bronchial washings did not yield $M$. chelonae in terms of bronchoscopist performing the procedure, date, nurse assistant, age of disinfecting solution, and time elapsed since the bronchoscope was cleaned.

The systematic search for a possible source of contamination led to the suction channel of four different bronchoscopes when all samples of rinsing fluid yielded $M$. chelonae. Cultures of tap water at the inlet of the washing machine and specimens from the tank of the washing machine were all negative for $M$. chelonae. The bronchoscopes were all examined by air leak test, but no puncture damage was found.

After this survey, the bronchoscope disinfection procedure was modified by extensive suctioning and rinsing of the suction channel with $70 \%$ alcohol immediately after machine disinfection. No additional episodes of cross-contamination or infection have occurred, and surveillance cultures were negative for $M$. chelonae in the next 12 month period. Follow-up cultures of patients in this episode have been negative, and no one has developed disease with $M$. chelonae.

\section{Discussion}

The flexible fibreoptic bronchoscope has been widely used for diagnosis and therapy of pulmonary diseases. However, transmission of pathogenic bacteria by improperly disinfected bronchoscopes can be a problem. Contamination of bronchoscopes with Proteus species [11], Klebsiella pneumoniae [12], or Pseudomonas aeruginosa [13] has been reported. From some of the contaminations, pulmonary infection can develop. Transmission of $M$. tuberculosis by contaminated bronchoscope, resulting in active disease, has also been documented [14]. Mycobacterial disease manifesting as a nosocomial outbreak which is bronchoscopy-related is often pseudoepidemic, i.e., "real clustering of false infection" [15]. Several episodes of pseudoepidemic caused by $M$. chelonae have been reported [16-21]: one episode came from contamination of a damaged suction channel [16], three outbreaks were caused by contaminated automated bronchoscope disinfection machines [17-19]. In this pseudoepidemic episode, the bronchoscopic suction channel was the possible source of contamination.

A review of the Hospital's laboratory records showed that the isolation of $M$. chelonae had been quite rare, occurring in fewer than 10 specimens annually. Most commonly, it came from specimens of eye or soft tissue infection. With the exception of this episode, no positive culture for $M$. chelonae was found from bronchial washing. The alertness both of clinicians and laboratory personnel noted the unusual increase in frequency of isolation of M. chelonae from specimens of bronchial washings. After thorough investigation, this episode proved to be a pseudoepidemic. According to the study of Weinstein and Stamm [15], pseudoepidemics come from errors in specimen processing, surveillance artefacts and clinical misdiagnosis. Of these, errors in speci-men processing are most common, as demonstrated in this study, where contamination of the suction channel of the bronchoscope was the apparent cause of the outbreak.

The source of the original contamination is unclear. There are five possibilities: 1) tap water, because $M$. chelonae is a common isolate from tap water; 2) a source patient who had had bronchoscopy without culture for $M$. chelonae some time before the outbreak occurred and may have contaminated the bronchoscopes; 3) contaminated glutaraldehyde, because repetitive cycles of disinfection cause decreased bactericidal effect from dilution and age of the activated glutaraldehyde; 4) automatic disinfecting machine, due to difficulty of cleaning, which may become a reservoir of mycobacteria; 5) local anaesthetic or a damaged suction channel (even with a negative air leak test). Since the suction channels of all four bronchoscopes were contaminated, the most likely source of contamination was the tap water used to rinse the bronchoscope. However, M. chelonae could not be isolated from tap water nor the automated washing machine. To resolve such problems here, $70 \%$ alcohol was used to rinse and for extensive suctioning through the suction channel of the bronchoscope after disinfection by the automated bronchoscope disinfection machine, a method also suggested by other studies [19, 22]. STIN et al. [20] suggested installation of a $0.2 \mu \mathrm{m}$ filter for tap water used in the automated washing machine. No further isolates of $M$. chelonae have been identified in 12 months of surveillance.

It has recently been reported that restriction fragment length polymorphism (RFLP) analysis of mycobacterial deoxyribonucleic acid (DNA) can help to explore the possible mechanism of spread of the organism, as well as identifying outbreak associated cases [23]. However, it is still difficult to verify the real source of the original contamination.

Although M. chelonae pulmonary disease has not developed in any of the patients, all patients should be followed. Previous study has shown that $M$. chelonae can cause transient colonization as well as pulmonary infection in patients who have undergone bronchoscopy with contaminated equipment [16]. The patients who develop clinical diseases are immunocompromised hosts. According to the recommendation of The Research Committee of the British Thoracic Society: before performing bronchoscopy on an immunocompromised patient, the bronchoscope should be washed in detergent, soaked for 60 min in $2 \%$ alkaline glutaraldehyde and rinsed in sterile water (or alcohol) to avoid contamination with opportunistic environmental mycobacteria [22].

The pseudoepidemic of bronchoscopy-related mycobacterial disease may lead to: 1) unnecessary potential toxic 
Table 2. - Summary of recommended procedures for cleaning and disinfection of flexible bronchoscopes

1. Dismantle the valve accessories and thoroughly wash and brush all parts of the bronchoscopes in neutral detergent.

2. Autoclave removable, heat stable parts.

3. Fit bacterial filters to the mains water inlet.

4. Prolonged exposure of bronchoscope to $2 \%$ glutaraldehyde in immunocompromised patients.

5. Extensive suction and rinse the bronchoscope with $70 \%$ alcohol after disinfection or immediately before use.

6. Thorough drying with pressurized air before storage.

7. Storage in sterile environment.

8. Regularly clean and replace the tubing in the machine.

9. Periodically send an aliquot of bronchoscope rinsing fluid for surveillance microbiological study.

antituberculous therapy; 2) potential risk of pulmonary infection, especially in an immunocompromised patient; 3 ) unnecessary diagnostic procedures or delay in diagnosis, as occurred in this study; 4) time and energy consumed in the search for the source of the outbreak; and 5 ) waste of medical resources. However, bronchoscopes are difficult to disinfect, and suction valves, even biopsy forceps, have all been implicated in infection outbreaks. Two percent glutaraldehyde was thought to be effective for machine cleaning to eradicate $M$. tuberculosis where disinfection with ethylene oxide is not feasible [24, 25]. However, contact time and the temperature also influence the activity of sterilization $[22,24,25]$; the recommended times have ranged 10-60 min. [22, 24, 25]. Even then, some rapidly growing mycobacterial strains can survive in 2\% glutaraldehyde [18]. Prolonged exposure of activated glutaraldehyde may damage or at least shorten the life of a bronchoscope. Therefore, it is necessary to determine a balance point between safety, instrument availability and duration of life of the bronchoscope. Since automated washing and disinfection machines for bronchoscopes play an important role in large medical centres, an optimal protocol and good surveillance system should be established. Recommendations for cleansing and disinfection procedure are summarized in table 2 [18, 22]. Most important, however, is being alert to any possibility of pseudoepidemic because, even with strict infection control procedures, contamination is still possible.

\section{References}

1. Brown TH. The rapidly growing mycobacteria - Mycobacterium fortuitum and Mycobacterium chelonei. Infect Control 1985; 6: 283-288.

2. Wolinsky E. Nontuberculous mycobacteria and association diseases. Am Rev Respir Dis 1979; 119: 107-159.

3. Moore M, Freichs JB. An unusual acid-fast infection of the knee with subcutaneous abscess-like lesions of the gluteal region. J Invest Dermatol 1953; 20: 133-169.

4. Schaad UB, Volttler TP, McCracken GH, Nelson JD. Management of atypical mycobacterial lymphadenitis in childhood. A review based on 380 cases. J Paediatr 1979; 95: 356-360.

5. Pottage JC Jr, Harris AA, Trenholme GH, Levin S, Kaplan RL, Feczko JM. Disseminated Mycobacterium chelone infection: a report of two cases. Am Rev Respir Dis 1982; 126: 720-722.

6. Lowry PW, Jarvis WR, Oberle AD, et al. Mycobacterium chelonae causing otitis media in an ear, nose and throat practice. N Engl J Med 1988; 319: 978-982.

7. Safranek TJ, Jarvis WR, Carson LA, et al. Mycobacterium chelonae wound infections after plastic surgery employing contaminated gentian violet skin-marking solution. N Engl J Med 1987; 317: 197-201.

8. Lowery PW, Beck-Sague CM, Bland LA, et al. Mycobacterium chelonae infection among patients receiving high-flux dialysis in a hemodialysis clinic in California. J Infect Dis 1990; 161: 85-90.

9. Tsukamura M, Mizuno S, Murata H. A simple identification system of clinically important mycobacteria. Kekkaku 1982; 57: 335-342.

10. American Thoracic Society. Diagnosis and treatment of disease caused by nontuberculous mycobacteria. Am Rev Respir Dis 1990; 142: 940-953.

11. Weinstein HJ, Bone RC, Ruth WE. Contamination of a fiberoptic bronchoscope with a Proteus species. Am Rev Respir Dis 1977; 116: 541-543.

12. Bottone EJ. Trace Klebsiella outbreak to flexible bronchoscopy. Perspect Infect 1980; 78: 252-253.

13. Hussain SA. Fiberoptic bronchoscope-related outbreak of infection with Pseudomonas. Chest 1978; 74: 483.

14. Wheeler PW, Lancaster D, Kaiser AB. Bronchopulmonary cross-colonization and infection related to mycobacterial contamination of suction valves of bronchoscopes. J Infect Dis 1989; 159: 954-958.

15. Weinstein RA, Stamm WE. Pseudoepidemics in hospital. Lancet 1977; 2: 862-864.

16. Pappas SA, Schaaf DM, DiCostanzo MB, King FW, Sharp JT. Contamination of flexible fiberoptic bronchoscopes. Am Rev Respir Dis 1983; 127: 391-392.

17. Fraser VJ, Jones M, Murray PR, et al. Contamination of flexible fiberoptic bronchoscopes with Mycobacterium chelonae linked to an automated bronchoscope disinfection machine. Am Rev Respir Dis 1992; 145: 853-855.

18. Gubler JGH, Salfinger M, Graevenitz AV. Pseudoepidemic of nontuberculous mycobacteria due to a contaminated bronchoscope cleaning machine: report of an outbreak and review of the literature. Chest 1992; 101: 1245-1249.

19. Elston RA, Hay AJ. Acid-fast bacillus contamination of a bronchoscope washing machine. J Hosp Infect 1991; 19 (1): 72-73.

20. Stine TM, Harris AA, Levin S, Rivera N, Kaplan RL. A pseudoepidemic due to atypical mycobacteria in a hospital water supply. J Am Med Assoc 1987; 258: 809-811.

21. Nye K, Chadha DK, Hodgkin P, Bradley C, Hancox J, Wise R. Mycobacterium chelonei isolation from bronchoalveolar lavage fluid and its practical implication. $J$ Hosp Infect 1990; 16: 257-261.

22. Woodcock A, Campbell I, Collins JVC, Hanson P, Harvey $\mathrm{J}$, Corris P. Bronchoscopy and infection control. Lancet 1989; i: 270-271.

23. Bennett SN, Peterson DE, Johnson DR, Hall WN, RobinsonDunn B, Dietrich S. Bronchoscopy-associated Mycobacterium xenopi pseudoinfections. Am J Respir Crit Care Med 1994; 150: 245-250.

24. Hanson PJV, Collins JV. AIDS, aprons, and elbow grease: preventing the nosocomial spread of human immunodeficiency virus and associated organisms. Thorax 1989; 44: 778-783.

25. Ayliffe GAJ, Babb JR, Bradley CR. Disinfection of endoscopes. J Hosp Infect 1986; 7: 296-299. 